\title{
Rate and Type of Infections in Children with Nephrotic Syndrome
}

\author{
Doaa Youssef Mohammed*, Mona Shaaban Ali Selim, Ali Mohammed Abo Zeid and Mayy Abd \\ Alfattah Neemat-Allah
}

Faculty of medicine, Zagazig University, Ash Sharqiyah, Egypt

"Corresponding Authors: Dr. Doaa Youssef Mohammed, Faculty of medicine, Zagazig University, Ash Sharqiyah, Egypt, Tel: 201222839220; E-mail: Dody5176@yahoo.com

Received: 04 January 2018; Accepted: 02 February 2018; Published: 01 March 2018

\begin{abstract}
Introduction: Changes in immunity are complex in nephrotic syndrome, affecting the cellular and humoral balance of specific immunity which may impair response to infection, currently recognized that at least half of relapses are triggered by infection. We aimed to describe the accurate rate of infection and specific type of treatment in nephrotic syndrome cases admitted with activity to nephrology unit.

Subjects and methods: This is a cross-sectional, case control study performed among 90 cases admitted nephrotic syndrome with activity, they were two group; group I 60 admitted with infection and group II 30 nephrotic syndrome admitted without infection. They were evaluated for the activity of disease, type of infections, occurrence of complications and fate of therapy and routine laboratory tests were done.

Result: The most common type of infection was gastroenteritis (GE) (25\%) of infected cases, 2nd most common Then Urinary tract infection (UTI) (21.7\%), While Pneumonia represented (15\%) of infection. there was no significant pathogen in (61.66\%), the most common pathogen is E.coli (25\%), followed by Shigella (6.66\%), pneumococci $(3.33 \%)$, klebsiella $(1.66 \%)$ and proteus $(1.66 \%)$ of all infected cases. The most frequently used antibiotic before culture is Ampicillin, sulbactam followed by cefotaxime and after culture Cefepime, Meronem, ceftazidime and Vancomycin.

Conclusion: We concluded that $66.6 \%$ of admitted children with NS with relapse were due to infection, the most common type of infection is gastroenteritis, followed by Urinary tract infection, Viral infection represents high rate of infected cases and the most common type of bacteria after culture is E.coli, Shigella, pneumococci.
\end{abstract}

Keywords: Immunity; Nephrotic syndrome; Gastroenteritis; Urinary tract infection; Viral infection 


\section{Introduction}

Nephrotic syndrome is a disease affects the kidney exclusively and is marked typically by the effacement of podocyte foot processes without glomerular deposit or inflammatory lesion [1]. Children with NS acquire many acute complications some of which may be serious and fatal; as infections, venous thromboembolism (VTE), and AKI, when clinical reflictions of infection and VTE on nephrotic children are clear, the epidemiology and fate of AKI remain vague [2].

Without treatment, nephrotic child is more prone to mortality, mostly due to bacterial infection. Before era of corticosteroids and antibiotics, $40 \%$ of children died, with $50 \%$ of these losses due to infection [3]. Recently it is proved that at least $50 \%$ of activities in pediatric onset NS are stimulated by a viral upper respiratory tract infection; this may be due to non-specific host response to infection more than to virus itself or their antibody response.

Thus, other infections such as urinary tract infection (UTI), diarrhea, peritonitis and skin infections have also been implicated. Many researches have proved the role of UTI as an important trigger of relapse, and the cause of steroid resistance. Thus; many studies have found specific initiators of activates, targeted interventions have been shown to modify disease course [4]. Children with nephrotic syndrome who respond to therapy have an $80-90 \%$ possibility of having one or more activates. $50 \%$ of those that have activity are infrequent relapses and can be managed with short courses of prednisone [3]. Our aim was to describe the accurate rate of infection and specific type of treatment in nephrotic syndrome cases admitted with activity to nephrology unit.

\section{Subjects and Methods}

This is a cross-sectional, case control study performed among admitted nephrotic syndrome cases with activity to inpatient pediatric nephrology unit, Zagazig University Hospitals. 90 subjects participated in this study; these were selected from the date of starting the study in July 2016 up till fulfilling the sample size in October 2016, they were divided into two group I 60 admitted with infection and group II 30 nephrotic syndrome admitted without infection. This study was approved from the Ethical Committee in Faculty of Medicine, Zagazig University. Written informed consent was taken from the study subjects (infected group+ non infected group and their parents).

We concluded Admitted nephrotic cases with activity or relapse, Age ranged from birth to 18years, both sexes were included and all social and educational classes. We excluded; admitted cases with no activity, activity without admission and those who refused participation.

Participants were subjected to a questionnaire which specially designed to collect; Socio-demographic data including age, sex, Clinical data including: age of onset of nephrotic syndrome, duration of illness ,number of relapses, response to steroid, types and duration of any medication including specific treatment as regarding antibiotic and anti-viral treatment, supportive treatment. All cases with nephrotic syndrome were evaluated for the activity of disease, type of infections, occurrence of complications and fate of therapy. Laboratory tests were done including) CBC, serum creatinine, BUN, Total serum protein, serum albumin and CRP). 


\section{Statistical Analysis}

Data were then imported into Statistical Package for the Social Sciences (SPSS version 20.0) (Statistical Package for the Social Sciences) software for analysis.

\section{Results}

90 child with nephrotic syndrome were admitted with relapse in our nephrology unit during the period of our study; they were group A 60 cases (44 males, 16 females) admitted with infection their mean age $6.66 \pm 4.6$ years old, and group B 30 (21 males, 9 females) without infection with mean age $5.15 \pm 3$ years old. The most common type of infection was gastroenteritis (GE) (25\%) of infected cases and; [it was caused by E.coli (33\%), viral infection (40\%), Shigella (20\%), and no pathogen in (6.7\%) of gastroenteritis cases], 2nd most common Then Urinary tract infection (UTI) (21.7\%); [it was caused by E.coli (64.2\%), viral (23.1\%), no pathogen in (15.4\%), proteus (7.7\%) and klebsiella in (7.7\%)], While Pneumonia represented (15\%) of infection; [it was caused by viral infection (55.6\%), no pathogen in (33.3\%) and pneumococci in (11.1\%)], Upper respiratory tract infection (URTI) also represented $(15 \%)$ of cases, no pathogen in $(55.6 \%)$, viral infection in $(44.6 \%)$. We found also Peritonitis represented $(1.7 \%)$ of infection it was caused by viral infection. Other cases were admitted with more than one site of infection as shown in Table 1.

Table 1 also showed that there was no significant pathogen in (61.66\%), the most common pathogen was E.coli (25\%), followed by Shigella (6.66\%), pneumococci $(3.33 \%)$, klebsiella $(1.66 \%)$ and proteus $(1.66 \%)$ of all infected cases. We found leukocytosis in (28.3\%), Neutropenia in (26.7\%) and Lymphocytosis in (21.7\%) of infected cases combined lymphocytosis, neutropenia and negative CRP results in $(21.7 \%)$ of cases.

We reported that the most frequently used antibiotic before culture is Ampicillin, sulbactam followed by cefotaxime and after culture Cefepime, Meronem, ceftazidime and Vancomycin are the most frequently used Table 2, while we used antiviral therapy in 13 cases $21.7 \%$ of infected cases. all cases received cyclosporine were presented with active disease, While not all cases received azathioprine presented with active disease ; that means that cyclosporine precipitate infection Table 3. We found that (91.7\%) of infected group had fair out come and (8.3\%) of cases had unfavorable outcome as following: (5\%) of cases show Activity leading to renal failure, (1.7\%) of cases died from pneumonia and (1.7\%) of cases show thrombosis Table 4.

\begin{tabular}{|c|c|c|c|c|c|c|c|c|c|}
\hline \multirow{3}{*}{$\begin{array}{l}\text { Type of } \\
\text { infection }\end{array}$} & \multirow[t]{3}{*}{ No } & \multirow[t]{3}{*}{$\%$} & \multirow{3}{*}{$\begin{array}{l}\text { Sample of } \\
\text { culture }\end{array}$} & \multicolumn{6}{|c|}{ Result of culture } \\
\hline & & & & \multicolumn{2}{|c|}{-ve $N=13$} & \multicolumn{2}{|l|}{+ ve $N=21$} & \multicolumn{2}{|c|}{ Viral $N=26$} \\
\hline & & & & No & $\%$ & No & $\%$ & No & $\%$ \\
\hline \multirow[t]{2}{*}{ GE } & \multirow[t]{2}{*}{15} & \multirow[t]{2}{*}{25} & \multirow[t]{2}{*}{ Stools } & \multirow[t]{2}{*}{1} & \multirow[t]{2}{*}{6.7} & E.coli (5) & 33.3 & \multirow[t]{2}{*}{6} & \multirow[t]{2}{*}{40} \\
\hline & & & & & & Shigella (3) & 20 & & \\
\hline \multirow[t]{3}{*}{ UTI } & \multirow[t]{3}{*}{13} & \multirow[t]{3}{*}{21.7} & \multirow[t]{3}{*}{ Urine } & \multirow[t]{3}{*}{2} & \multirow[t]{3}{*}{15.4} & E.coli (6) & 46.2 & \multirow[t]{3}{*}{3} & \multirow[t]{3}{*}{23.1} \\
\hline & & & & & & Proteus (1) & 7.7 & & \\
\hline & & & & & & Klebsiella (1) & 7.7 & & \\
\hline Pneumonia & 9 & 15 & Sputum & 3 & 33.3 & Pneumococci (1) & 11.1 & 5 & 55.6 \\
\hline
\end{tabular}

Arch Clin Med Case Rep 2018; 2 (2): 31-39 


\begin{tabular}{|c|c|c|c|c|c|c|c|c|c|}
\hline URTI & 9 & 15 & Sputum & 5 & 55.6 & - & 0.0 & 4 & 44.4 \\
\hline Peritonitis & 1 & 1.7 & Bl. culture & - & 0.0 & - & 0.0 & 1 & 100 \\
\hline GE +UTI & 3 & 5 & Urine & - & 0.0 & E.coli (2) & 66.7 & 1 & 33.3 \\
\hline $\begin{array}{l}\text { Pneumonia+ } \\
\text { UTI }\end{array}$ & 2 & 3.3 & Urine & 2 & 100 & - & 0.0 & - & 0.0 \\
\hline $\begin{array}{l}\text { Pneumonia+ } \\
\text { Peritonitis }\end{array}$ & 2 & 3.3 & B1. culture & - & 0.0 & - & 0.0 & 2 & 100 \\
\hline GE + URTI & 1 & 1.7 & Stools & - & 0.0 & - & 0.0 & 1 & 100 \\
\hline $\begin{array}{l}\text { GE + } \\
\text { Pneumonia }\end{array}$ & 2 & 3.3 & Stools & - & 0.0 & Shigella (1) & 50 & 1 & 50 \\
\hline $\begin{array}{l}\text { GE + } \\
\text { Frunculosis }\end{array}$ & 1 & 1.7 & Stools & - & 0.0 & - & 0.0 & 1 & 100 \\
\hline GE + Peritonitis & 2 & 3.3 & Stools & - & 0.0 & E.coli (1) & 50 & 1 & 50 \\
\hline
\end{tabular}

GE: gastroenteritis; UTI: Urinary tract infection; URTI: Upper respiratory tract infection

Table 1: Types of infection and culture results in infected group.

\begin{tabular}{|c|c|c|c|}
\hline & & Frequency & Percent \\
\hline \multirow{12}{*}{ 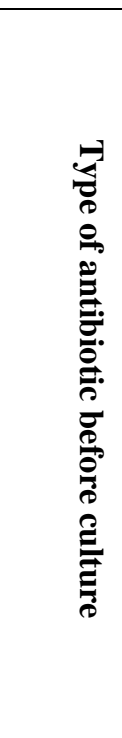 } & No & 19 & 31.7 \\
\hline & Cefepime & 2 & 3.4 \\
\hline & Cefoperazone (Cefobid ) & 8 & 13.3 \\
\hline & Cefotaxime (Cefotaxim) & 1 & 1.7 \\
\hline & Ceftriaxone & 2 & 3.3 \\
\hline & Cefotaxime (Claforan) & 19 & 31.6 \\
\hline & ceftazidime(Fortum) & 1 & 1.7 \\
\hline & (Ampicillin, sulbactam) unasyn & 26 & 43.3 \\
\hline & Unasyn and Cefobid & 1 & 1.7 \\
\hline & Vancomycin & 5 & 8.3 \\
\hline & (azithromycin) Zithrodose & 1 & 1.7 \\
\hline & (azithromycin) Zithromax & 1 & 1.7 \\
\hline \multirow{7}{*}{ 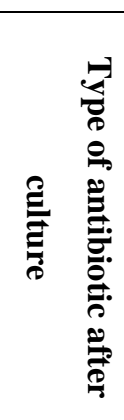 } & No & 57 & 95.0 \\
\hline & Cefepime & 1 & 1.7 \\
\hline & Meropenem (Meronem) & 1 & 1.7 \\
\hline & Meronem & 1 & 1.7 \\
\hline & ceftazidime (Fortum) & 1 & 1.7 \\
\hline & Vancomycin & 1 & 1.7 \\
\hline & Total & 60 & 100.0 \\
\hline
\end{tabular}

Table 2: Antibiotic therapy used to treat infection. 


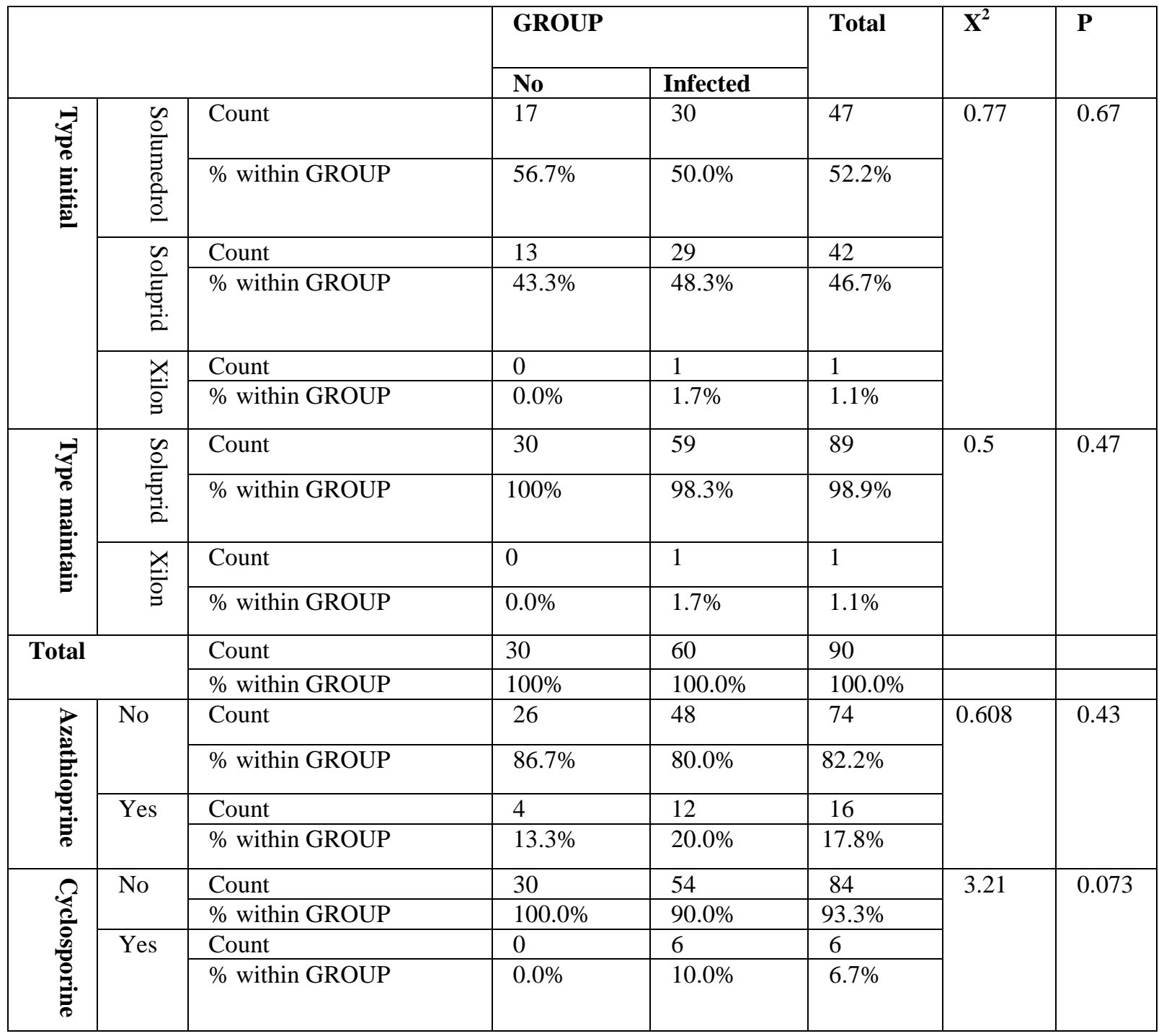

Table 3: Types of steroid and other immunosuppressant used to induce remission.

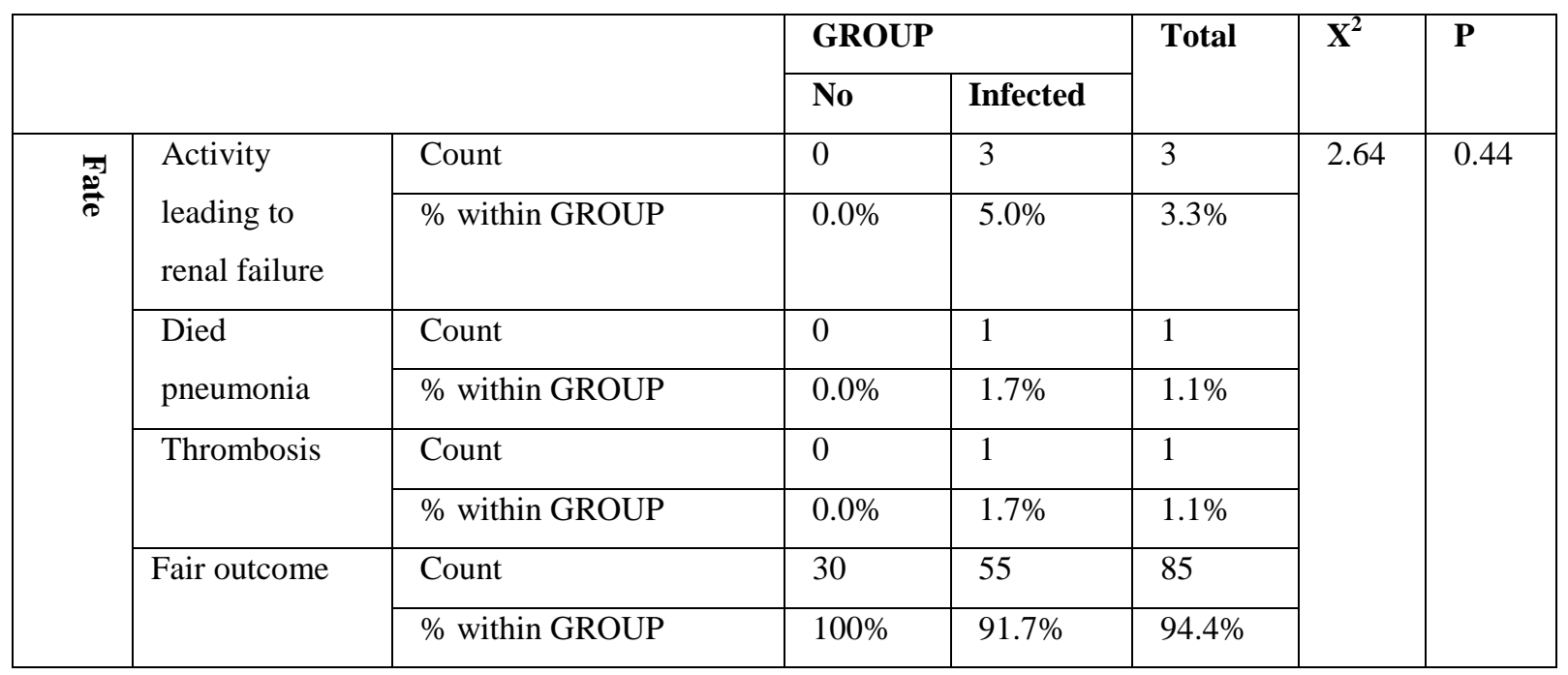




\begin{tabular}{|c|c|c|c|c|c|c|c|}
\hline \multirow{4}{*}{ 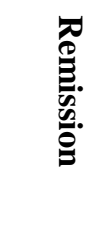 } & Unfavorable & Count & 0 & 5 & 5 & \multirow[t]{4}{*}{1.29} & \multirow[t]{4}{*}{0.25} \\
\hline & & $\%$ within GROUP & $0.0 \%$ & $8.3 \%$ & 5.5 & & \\
\hline & \multirow[t]{2}{*}{ Favorable } & Count & 30 & 55 & 85 & & \\
\hline & & $\%$ within GROUP & $100 \%$ & 91.7 & 94.5 & & \\
\hline \multirow[t]{2}{*}{ Total } & & Count & 30 & 60 & 90 & - & - \\
\hline & & $\%$ within GROUP & $100.0 \%$ & $100.0 \%$ & $100.0 \%$ & - & - \\
\hline
\end{tabular}

Table 4: Fate and remission rate in comparison between infected and non-infected group.

\section{Discussion}

Episodes of nephrotic syndrome are often temporally associated with occurrence of infection. As glucocorticoids and other immuno-suppressive drugs is the mainstay of therapy, infection occurring during therapy is well-known and is partially explainable by the relative immunocompromised state. However, infection often occurs even when the child is not on glucocorticoid therapy [5], Webb and his colleagues 2016 reported that awareness of common pathogens responsible for infections in children with INS is vital to guide initial antimicrobial therapy [6].

The current study showed that there is high rate of infection among admitted cases with activity (66.6\%), and we found the high incidence of infection due to admission of cases during summer with high rate of gastroenteritis and inclusion of URTI. Other studies reported different rates of infection in NS cases; for example [7] Krishnan, 2017 mentioned that a total of 246 children with nephrotic syndrome were enrolled, of whom 46 children developed 48 (19.6\%) episodes of infections. Ajayan et al. [8] mentioned that Major infections were defined as; those that are scattered, hit deep organs, need hospitalization or potentially fatal. The incidence of these infections was $36.6 \%$. According to Soares et al. [9] There was a significant decrease in the incidence and transmission of infection nowadays in Brazil because of the wider vaccination administration to children. This may explain less incidence of this infection as a cause of NS; While Alwadhi et al. [5] reported that of the 76 activities, 8 were excluded from analysis. Of the remaining 68 nephrotic attacks in 60 patients, there was clue of infection in 57 attacks (83.8\%).

In our study; The most common type of infection was gastroenteritis (GE) (25\%) of infected cases and 2nd most common Then Urinary tract infection (UTI) (21.7\%), While Pneumonia represented (15\%) of infection. According to Uwaezuoke et al. [4] and Krishnan et al. [7] Pneumonia (41.7\%) was the most common infection, urinary tract infection (25\%), septicemia (16.7\%), followed by spontaneous bacterial peritonitis (8.3\%), Ajayan et al. [8] mentioned that within the major infections, peritonitis plus pneumonia were for $72.9 \%$, while urinary tract infections plus cellulitis were $16.2 \%$. In a U.S. retrospective study focusing on 1ry peritonitis in INS, S. pneumoniae was the most common organism isolated, about for $38 \%$ of patients, with Gram-negative bacteria only $3 \%$ of patient. And $27 \%$ of patients were with negative culture results. Another article studying peritonitis in childhood NS found that S. pneumoniae was the most common pathogen identified (50\%), but that Escherichia coli accounted for $25 \%$ of cases, and $16 \%$ of cases were culture-negative [6].

Our study also showed that there was no significant pathogen in (61.66\%), the is E.coli (25\%), followed by Shigella (6.66\%), pneumococci (3.33\%), klebsiella (1.66\%) and proteus (1.66\%) Of all infected cases, matching our results Alwadhi et al. [5] mentioned that of [13] UTI, [11] had Gram negative organisms, namely Escherichia-coli 
and Klebsiella. Enterococcs was isolated in the other two. Urinary tract infection was asymptomatic in (77\%) of cases. Three out of nine cases of peritonitis showed Gram negative bacilli on staining of peritoneal fluid examination. However, no organism was isolated from any ascetic fluid samples. Our study showed that there was high statistical significant difference as regarding CRP results between infected and non-infected group before and after treatment. And show that $(60 \%)$ of CRP results in infected group are negative that explain culture results that no pathogen in $(66.6 \%)$ be viral infection; Therefore, sCRP may serve as a reliable marker of inflammation in this setting according to Wasilewska et al. [10] Increases in CRP values are non-specific and should not be interpreted without a complete clinical evaluation. Conventional assays for CRP are not sensitive enough and, therefore, not reliable in healthy subjects Conventional CRP assays are indicated for use for evaluation of infection, the children with a relapse of nephrotic syndrome showed a significant increase in -CRP level.

In our study, 36/60 (60\%) of infected cases had CRP negative and 40\%were CRP positive. As regarding TLC 26.7\% were neutrophilic and $21.7 \%$ show Lymphocytosis. As regarding culture results 37/60 (61.6\%) of cases show no growth; so $(60 \%)$ of patients had viral infection. According to Uwaezuoke et al. [4]. It is found that at least half of activities were triggered by a viral upper respiratory tract infection which may be associated to non-specific host response to infection. In our study that there was statistical significant affection of hemoglobin and serum creatinine in infected than non-infected group. Also, there is no significant difference between both groups regarding steroid response whether infected or not.

Also, in our study No statistical significant differences as regarding type of steroid used to induce remission, According to Larkins et al. [11] the majority of children who relapse continue to respond to steroids throughout their subsequent course, and the long-term prognosis, including maintenance of normal kidney function, is good, While, Gulati et al. [12] mentioned that daily doses of steroid, during inter-current infections, significantly decrease activity frequencies and number of children with frequently relapsing NS. The decline in activity times in the study was mainly because of decreasing number of infection-triggered relapses. $70 \%$ of activities in the intervention patients and $74 \%$ in the controls were preceded by infections, mainly of the upper respiratory tract. The mechanism by which infections induce activities of NS is vague but might be due to the up-regulation of T cells plus cytokine induced rise in proteinurea. But Jamin et al. [13] mentioned that the clinical course of INS is largely dependent upon the response to steroids. Relapses are frequent after withdrawal of steroid treatment, with up to $60 \%$ of patients being steroiddependent.

In our study, all cases receiving cyclosporine presented with active disease. While not all cases receiving azathioprine presented with active disease that means that cyclosporine precipitate infection. According to Soares et al. [9] immunosuppressant in children with chronic infections may lead to the deterioration of the infection and of the general clinical state. So; most regimes of management of children with NS suggest screening of infections, because these may need specific treatment. According to Uwaezuoke et al. [4]. The new findings however do not preclude the requirement for the use of chemotherapy drugs like steroids or cycholsporins as these drugs also alter podocyte function as well as structure. So , the KDIGO recommendation of prescribing daily steroids in activities of upper respiratory infections still a golden intervention targeting reduction the risk of activity. 
In our study the most frequently used antibiotic before culture is Ampicillin, sulbactam followed by cefotaxime and after culture Cefepime, Meropenem, ceftazidime and Vancomycin are the most. There is high statistical significant difference between both groups regarding antiviral response between infected and non-infected group. There was (21.7\%) of infected cases received antiviral treatment, According to Yildirim et al. [14] it appears that penicillin, ampicillin, or cefuroxime should be adequate to treat children hospitalized with pneumonia due to pneumococcal isolates .Oral therapy with amoxicillin, amoxicillin-clavulanate, or cefuroxime also should be effective for 1st outpatient management or when ending therapy once the child has resolution of signs and symptoms following parenteral treatment. While, Hogan et al. [15] stated that Antiviral treatment leads to a complete remission of the NS, with no requirement for steroid.

In our study, there is high significant difference between both groups regarding plasma transfusion between infected and non-infected group and no statistical significant difference regarding albumin infusion this comes into agreement with Alwadhi et al. [5] who reported that Children with infection had significantly lower serum albumin compared to non-infected children.

In our study there is $(91.7 \%)$ of infected group had fair out come and $(8.3 \%)$ of cases had unfavorable outcome as following: (5\%) of cases show Activity leading to renal failure, (1.7\%) of cases died from pneumonia and (1.7\%) of cases show thrombosis. Krishnan et al. [7] Mentioned that there was no mortality in his study. While Uwaezuok et al. [4] reported that only $20 \%$ respond to the medication with a more risk of developing end-stage renal failure. Lin et al. [16] reported that the long-term prognosis of NS is mostly good; but, its characteristics and related treatment can greatly affect childhood development and quality of life in those patients. Resistant cases in some patients may deteriorate up to end-stage renal disease. Larkins et al. [11] Reported that without therapy, NS in children comes with high risk of mortality, especially of bacterial infection. In the past before the use of steroid and antibiotics, $40 \%$ of patients were lost, with $50 \%$ of these losses were due to infection.

\section{Conclusion}

We concluded that $66.6 \%$ of admitted children with NS with relapse were due to infection, the most common type of infection is gastroenteritis, followed by Urinary tract infection, upper respiratory tract infection and Chest infection. We concluded also that Viral infection represents high rate of infected cases depending on clinical and laboratory data and the most common type of bacteria after culture is E.coli, Shigella, pneumococci, klebsiella and proteus..

\section{References}

1. Jamin A, Dehoux L, Dossier C, et al. Toll-like receptor 3 expression and function in childhood idiopathic nephrotic syndrome, Clin Exp Immunol 182 (2015): 332-345.

2. Rheault MN, Zhang L, Selewski DT, et al. Midwest Pediatric Nephrology Consortium, AKI in Children Hospitalized with Nephrotic Syndrome. Clin J Am Soc Nephrol 10 (2015): 2110-2118.

3. Kidney International Supplements. Steroid-sensitive nephrotic syndrome in children. Kidney Int Suppl 2 (2012): 163-171.

4. Uwaezuoke SN. Steroid-sensitive nephrotic syndrome in children: triggers of relapse and evolving hypotheses on pathogenesis, Ital J Pediatr 41 (2015): 19. 
5. Alwadhi RK, Mathew JL, Rath B. Clinical profile of children with nephrotic syndrome not on glucorticoid therapy, but presenting with infection. J Paediatr Child Health 40 (2004): 28-32.

6. Webb NJ, Frew E, Brettell EA, et al. Short course daily prednisolone therapy during an upper respiratory tract infection in children with relapsing steroid-sensitive nephrotic syndrome. Trials 15 (2014): 147.

7. Krishnan C, Rajesh TV, Shashidhara HJ, et al. Major infections in children with nephrotic syndrome International Journal of Contemporary Pediatrics 4 (2017): 346-350.

8. Ajayan P, Krishnamurthy S, Biswal N, et al. Clinical spectrum and predictive risk factors of major infections in hospitalizedchildren with nephrotic syndrome. Indian Pediatr 50 (2013): 779-781.

9. Soares SF, Donatti TL, Souto FJ. Serological markers of viral, syphilitic and toxoplasmic infection in children and teenagers with nephrotic syndrome: Rev Inst Med Trop Sao Paulo 56 (2014): 499-504.

10. Wasilewska A, Zoch-Zwierz W, Tobolczyk J, et al. High-sensitivity C-reactive protein (hs-CRP) level in children with nephrotic syndrome. Pediatr Nephrol 22 (2007): 403-408.

11. Larkins N, Kim S, Craig J, et al. Steroid-sensitive nephrotic syndrome: an evidence-based update of immunosuppressive treatment in children. Arch Dis Child 101 (2005): 404-408.

12. Gulati A, Sinha A, Math A et al. Daily Corticosteroids Reduce Infection-associated Relapses in Frequently Relapsing Nephrotic Syndrome: A Randomized Controlled Trial, Clin J Am Soc Nephrol 6 (2011): 63-69.

13. Jamin A, Dehoux L, Dossier C, et al. Toll-like receptor 3 expression and function in childhood idiopathic nephrotic syndrome, Clin Exp Immunol 182 (2015): 332-345.

14. Yildirim I, Shea KM, and Pelton S. Pneumococcal Disease in the Era of Pneumococcal Conjugate Vaccine Infect Dis Clin North Am 29 (2015): 679-697.

15. Hogan J, Fila M, Baudouin V, et al. Cytomegalovirus infection can mimic genetic nephrotic syndrome: a case report 22 (2015): 156.

16. Lin JN, Lin CL, Yang CH, et al. Risk of Nephrotic Syndrome following Enteroviral Infection in Children: A Nationwide Retrospective Cohort Study. PLoS One (2016): 0161004.

Citation: Doaa Youssef Mohammed, Mona Shaaban Ali Selim, Ali Mohammed Abo Zeid and Mayy Abd Alfattah Neemat-Allah. Rate and Type of Infections in Children with Nephrotic Syndrome. Archives of Clinical and Medical Case Reports 2 (2018): 38-46.

\footnotetext{
(C)
Creative Commons Attribution (CC-BY) license 4.0
} 